\title{
Recognizing Driver Braking Intention with Vehicle Data Using Unsupervised Learning Methods
}

\section{Yang Xing, Chen Lv, and Wang Huaji}

Cranfield University

\section{Hong Wang}

University of Waterloo

\section{Dongpu Cao}

Cranfield University

CITATION: Xing, Y., Lv, C., Huaji, W., Wang, H. et al., "Recognizing Driver Braking Intention with Vehicle Data Using Unsupervised Learning Methods," SAE Technical Paper 2017-01-0433, 2017, doi:10.4271/2017-01-0433.

\begin{abstract}
Recently, the development of braking assistance system has largely benefit the safety of both driver and pedestrians. A robust prediction and detection of driver braking intention will enable driving assistance system response to traffic situation correctly and improve the driving experience of intelligent vehicles. In this paper, two types unsupervised clustering methods are used to build a driver braking intention predictor. Unsupervised machine learning algorithms has been widely used in clustering and pattern mining in previous researches. The proposed unsupervised learning algorithms can accurately recognize the braking maneuver based on vehicle data captured with CAN bus. The braking maneuver along with other driving maneuvers such as normal driving will be clustered and the results from different algorithms which are K-means and Gaussian mixture model (GMM) will be compared. Additionally, the importance evaluation of features from raw dataset respect to driving maneuvers clustering will be proposed. The experiment data are collected from a pure electric vehicle in real world. Final results show that the proposed method can detect driver's braking intention in a very beginning moment with a high accuracy and the most important features for driving maneuver clustering are selected.
\end{abstract}

\section{Introduction}

As one of the most important control area of the vehicle, longitudinal vehicle control system has been widely studied in the past decades. A variety of commercial products such as adaptive cruise system (ACC) [1] and forward collision assistance system $[\underline{2}-\underline{3}, \underline{15}]$ have been extensively developed. However, previous products either use vehicle state data only or lack of interaction with drivers. Without taking driver into consideration, these systems are less likely to communicate with driver efficiently or even annoy the driver. To develop a higher level interaction ability driver braking assistance system, it is important to take driver status into consideration.
There are many researches focusing on the design of intelligent driver assistance and braking systems to incorporate with driver $[\underline{4}, \underline{5}, \underline{6}, \underline{7}$, $\underline{8}, \underline{9}, \underline{10}]$. In [11], an adaptive longitudinal driving assistance system include adaptive cruise control and forward collision avoidance system considering driver behavior and characteristic is proposed and a recursive least square self-learning algorithm for driver characteristic modeling is introduced. In [12], a lane departure avoidance system is applied to prevent the driver from inattention lane change based on the combination of lateral active steering controller and longitudinal differential braking controller. In [13], this paper studies driver braking intention on the testbed of commercial vehicles with multiple drivers. The authors predict driver braking intention based on a 3-D computational model, which using pedal displacement and its change rate as inputs, and the output is braking intensity. The proposed model can precisely identify emergency and non-emergency braking. In [14], a method of building pedestrian automatic emergency braking system is introduced based on two model cars with multiple kinds of sensors. Three different levels of deceleration are proposed to model the braking curve of the tested vehicle. In [25], two machine learning methods which are support vector machine and hidden Markov model are introduced to classify driver brake behavior at intersections. The authors classified driver style as compliant and violating according to whether the drivers will brake safely according to the signs at intersection or not. The proposed system achieved $80 \%$ accuracy for driver behavior classification at intersection. The authors in [16] proposed a braking predictive system which not only identify the necessity of braking action but also able to determine whether the driver has braking intention or not. Driver braking intention is represented with probabilities generated by Bayesian framework. Data are captured from real-world vehicle with multi-modal data format such as CAN bus data, foot and head dynamics, etc. The braking prediction model is trained to be able to predict driver braking intention up to one and a half second earlier. Research in [17] focused on the analysis of car 
following and the braking characteristic of expert drivers. The braking pattern of expert drivers will be performed according to proposed index and the applied to the braking assistance system.

In addition to the aforementioned researches which focus on predict driver braking intention with either driver behaviors or vehicle information, some researchers also try to use electroencephalograph (EEG) signals to identify driver intention directly. Kim J W and Haufe [18] aim to prove the ability of neural correlated electrophysiology to improve the prediction of emergency braking situations in real world driving environment. Vehicle parameters as well as EEG, EMG signals were used to train the classification system. A regularized linear discriminant classifier was trained to identify emergency braking intention. Their conclusion suggested that electrophysiology method can be efficiently used in braking assistant system. Haufe [19] use EEG and electromyography (EMG) as input signal to make prediction of driver's emergency braking intent. The signals after feature extraction procedure were fed into regularized linear discriminant analysis classifier. The simulated systems with EEG showed a $130 \mathrm{~ms}$ earlier detection than those only rely on brake pedal signals.

Human driver intention recognition is a difficult task due to it is a highly random signal and unable to measure directly at this moment. It can only be inferred with outer human behaviors or the indirectly measured brain signals. Therefore, in previous research, most of relative research focus on using machine learning methods especially supervised learning to train an intention classifier. However, one of the big challenge of using supervised learning methods is hard to define the true intention label. In contrast, unsupervised learning is suitable for those data that we do not know exactly their labels. Hence, it can be used to cluster the data to the group it most likely belongs to and able to find the intrinsic pattern in the data. In this paper, instead of identifying human intention with supervised learning methods, we use unsupervised learning method to recognize the braking intention. In addition, we compare two different unsupervised learning methods which are K-means and Gaussian Mixture Model to evaluate their different performance on braking intention recognition task. Finally, we also evaluate the contribution of different features to intention recognition, and find out the key features.

This paper is organized as follow: Section 2 present the basic background of unsupervised learning methods used in this paper. In section 3, the experimental environment and design strategy are discussed briefly. Section 4 illustrate the braking intention identification result of the proposed methods, and features used to train the model has been compared. Finally, section 5 developed the discussion of the research.

\section{Unsupervised Learning Background}

In this part, the basic background of two unsupervised learning methods known as K-means and Gaussian Mixture Model that used to identify human driver braking intention are introduced. The reason of using unsupervised learning methods is because human intention is difficult to be labeled unless the experiment is carefully designed. In the following sections, we try to analysis driver braking intention with vehicle CAN bus only and using unsupervised learning methods is selected to find the cluster information. Since these two methods has been widely studied, in this part, only the key ideas are mentioned.

\section{K-Means}

$\mathrm{K}$-means algorithm [21] is a popular unsupervised machine learning method which has been widely used in previous research for data clustering. The basic idea behind K-means algorithm is to minimize the distance between data points and the proposed cluster centers. One of the most important aspects of K-means algorithm is distance measurement between data point and there are various methods can be used. Among them, the most two common ways which are known as Euclidean distance and Manhattan distance are illustrated below:

Euclidean distance:

$$
D_{i, j}=\sqrt{\sum_{q=1}^{n}\left|v_{i, q}-v_{j, q}\right|^{2}}
$$

Manhattan distance:

$$
D_{i j}=\sum_{q=1}^{n}\left|v_{i, q}-v_{j, q}\right|
$$

Where $i$ and $j$ are two n-dimensional data points denoted as $\mathrm{v}$, where $v_{i}=\left(v_{i 1}, v_{i 2} \cdots, v_{i, n}\right)$ and $v_{j}=\left(v_{i 1}, v_{j 2}, \cdots, v_{j n}\right)$, and $D_{i, j}$ represents the distance between two data points.

The object of K-means algorithm is to minimize the distance between data and their cluster center in each group. Table 1 shows the process of K-means algorithm.

Table 1. K-means algorithm process

K-means Algorithm
Inputs: data, cluster number K
Process:
1. Random select the K positions as the center of K groups.
2. Assign cluster centers to these K position.
3. For each data, compute the distance between the data and the
4. $\quad$ Assign xi to the cluster with minimum distance.
5. For each cluster center, move the cluster center to the mean
6. $\quad$ If stop criteria meet, stop, otherwise jump to step 2 and repeat.

In step 6, the stopping criteria can be either pre-determined loop limitation or an optimal status is achieved. In that case, the stopping criterion is a minimum value achieved between the distance of data point and the cluster center within same group, therefore, the objective of K-means is to:

$$
\min \sum_{i=1}^{K} \sum_{x \in C_{i}} \operatorname{dist}\left(c_{i}, x\right)^{2}
$$

Where $\mathrm{K}$ is the total number of cluster centers selected in beginning, $x$ are the data points belongs to cluster $C_{i}$ and $C_{i}$ is the cluster center for each group. 


\section{Gaussian Mixture Model}

Unlike K-means which minimize the distance directly, data distribution in GMM [르] [르 ] are viewed as the output of a mixture of probability density function. Specifically, the probability distribution of the points can be generated by a mixture of Gaussian function. GMM can be viewed as a soft version of K-means since it uses probability to measure the similarity between points rather than direct distance measurement. Through training the model with these points, we can obtain the distribution of data and handle the uncertainty among them.

The multivariate Gaussian Mixture model can be represented as:

$$
N(x \mid \mu, \Sigma)=\frac{1}{(2 \pi)^{d / 2} \Sigma^{1 / 2}} \exp \left(-\frac{1}{2}(x-\mu)^{T} \Sigma^{-1}(x-\mu)\right)
$$

Where $\mu$ and $\sum$ are the mean and covariance of the multivariate Gaussian function.

The probability distribution of the data given by GMM can be shown as:

$$
p(x)=\sum_{k=1}^{K} \pi_{k} N\left(x \mid \mu_{k}, \sum_{k}\right)
$$

where $\mathrm{K}$ is the number of mixture components, $\pi_{k}$ is weight of each Gaussian density function which should meet the normalization and positive requirements:

$$
\sum_{k=1}^{K} \pi_{k}=1
$$

And

$$
0 \leq \pi_{k} \leq 1
$$

The GMM maximum likelihood can be estimated with ExpectationMaximization (EM) algorithms, the detail information of EM can be found in [므].

\section{Experiment Design}

In this part, the key parameters of the testbed electric vehicle and driving cycle are illustrated. Then, 12 most relevant parameters which are relevant to braking behaviors are selected from CAN bus signals. Finally, according to the selected parameters, the unsupervised learning methods mentioned above are used to figure out the hidden braking rules that behind the data.

\section{Case-Study Vehicle}

The case-study vehicle of this work is selected as a pure electric passenger car, which has the most typical powertrain configuration. The car is driven in front axle by a permanent magnet synchronous motor, which can work in two states as a driving motor or a generator. The battery is electrically connected with the motor, and it can be discharged or charged for motoring or absorbing the regenerative

\begin{tabular}{|c|c|c|c|}
\hline & Parameter & Value & Unit \\
\hline \multirow{4}{*}{ Vehicle } & Total mass $(m)$ & 1360 & $\mathrm{~kg}$ \\
\hline & Wheel base $(L)$ & 2.50 & $\mathrm{~m}$ \\
\hline & Frontal area $(A)$ & 2.40 & $\mathrm{~m}^{2}$ \\
\hline & Air resistance coefficient $\left(C_{D}\right)$ & 0.32 & - \\
\hline \multirow{3}{*}{ Electric motor } & Rated power & 20 & $\mathrm{~kW}$ \\
\hline & Peak power & 45 & $\mathrm{~kW}$ \\
\hline & Maximum torque & 144 & $\mathrm{Nm}$ \\
\hline \multirow{2}{*}{ Battery pack } & Voltage & 326 & V \\
\hline & Capacity & 66 & $\mathrm{Ah}$ \\
\hline \multirow{2}{*}{$\begin{array}{c}\text { Transmission } \\
\text { and final drive }\end{array}$} & Final drive ratio & 3.79 & - \\
\hline & Transmission ratio & 2.10 & - \\
\hline
\end{tabular}
power during driving processes respectively. Some key parameters of the case study vehicle are listed in Table 2.

Table 2. Key parameters of the case-study electric vehicle.

\section{Driving Cycle}

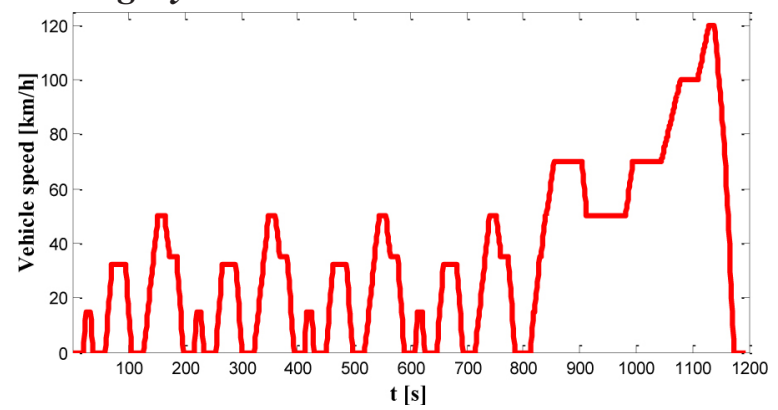

Figure 1. Road test under the NEDCE driving cycle.

To further the study of the impacts of unsupervised learning methods on driver braking intentions, vehicle tests care carried out on chassis dynamometer under typical driving cycles. In this experiment, we use the New European Drive Cycle (NEDC) as testing protocol. NEDC is a combination of the European Union Urban Driving Cycle (ECE) and the Extra Urban Driving Cycle (EUDC). It has been widely used for the electric vehicle energy consumption and regenerative braking performance testing. As shown in Figure 1, a complete NEDC driving cycle contains four repeated ECE driving cycles following with an EUDC section to exhibits a high way driving speed pattern with highest speed of $120 \mathrm{~km} / \mathrm{h}$.

\section{Parameter Selection}

Selecting the most relevant parameters and features is the primary task for unsupervised clustering. Driver baking behavior belongs to the scope of longitudinal behavior, therefore, to recognize driver's braking maneuver, the longitudinal vehicle parameters are selected and chosen from the CAN bus. The brake pressures of four wheels are captured separately, battery SOC and current is used to test whether varying battery status influence the detection result [24]. In this part, we choose 12 parameters from CAN bus, the detailed information is shown in Table 3 below. 
Table 3. Selected CAN bus signals for model training.

\begin{tabular}{|c|c|c|c|}
\hline No. & Signal Name & No. & Signal Name \\
\hline 1 & $\begin{array}{c}\text { Command Motor torque } \\
(\mathrm{N} \cdot \mathrm{m})\end{array}$ & 7 & Motor speed $(\mathrm{rad} / \mathrm{s})$ \\
\hline 2 & $\begin{array}{c}\text { Left front brake pressure } \\
(\mathrm{MPa})\end{array}$ & 8 & Acceleration pedal position \\
\hline 3 & $\begin{array}{c}\text { Right front brake pressure } \\
(\mathrm{MPa})\end{array}$ & 9 & $\begin{array}{c}\text { Left front wheel speed } \\
(\mathrm{rad} / \mathrm{min})\end{array}$ \\
\hline 4 & $\begin{array}{c}\text { Left rear brake pressure } \\
(\mathrm{MPa})\end{array}$ & 10 & $\begin{array}{c}\text { Right front wheel speed } \\
(\mathrm{rad} / \mathrm{min})\end{array}$ \\
\hline 5 & $\begin{array}{c}\text { Right rear brake pressure } \\
(\mathrm{MPa})\end{array}$ & 11 & Battery SOC $(\%)$ \\
\hline 6 & Velocity $(\mathrm{km} / \mathrm{h})$ & 12 & Batter Current $(\mathrm{A})$ \\
\hline
\end{tabular}

\section{Unsupervised Clustering Training Process}

One of the most important parameter for unsupervised clustering methods is cluster number which need to be chosen before learning. However, since we do not know the exactly number of cluster centers, we choose a set of numbers to evaluate and compare the performance. In the following part, the quantity of cluster centers ranging from two to five are applied to K-means and Gaussian mixture model separately. In addition to the algorithm evaluation, we also examined which signals are the most correlated factor for unsupervised clustering.

\section{Experiment Result}

In this section, the clustering performance for both K-means and GMM are illustrated with cluster centers ranging from two to five. Then, the correlation coefficients are calculated to help determine the most important features.

\section{K-Means Result}

From the clustering result of K-means, as shown in Figure 2, the braking intention can be detected successfully with different cluster numbers.

In Figure 2, the $\mathrm{x}$-axis is time with unit in second, the y-axis is the scaled values of the input signals, all the signals have been rescaled between 0 and 1 . The blue line represents for labels given by $\mathrm{K}$-means, red line is the left front brake pressure, and green line represents for the velocity of vehicle. To better visualize the result, we chose the first 200 points of the data, a complete result can be found in Appendix. Besides, since the value for all the factors has been scaled between 0 to 1 , the label given by K-means also be divided by the relative cluster number for better visualization.

From Figure 2 it can be observed that the braking process (when red line beyond zero) can be grouped correctly with different cluster numbers. For example, in the first image, once braking happens, which means a brake pressure is detected, the cluster label changed. As the number of cluster center increase, the cluster become more complex and there are more scenarios can be detected. As shown in the bottom image which is generated by five cluster centers, the braking intention can be detected. Besides, the label also changes as vehicle velocity varies. K-means with four cluster centers divided the driving cycle into four different scenarios which are acceleration, constant velocity driving, deceleration and stopping process. Due to we only show the first 200 steps, labels given by K-means with 4 or 5 cluster centers may be incomplete. A detail result is shown in Appendix. From above result we can see that once the braking occurs, the label change accordingly. For most cases, the braking maneuver will last for a short period, once the label changes to the braking group, it can be viewed as the driver is going to decelerate the vehicle in the coming short period and hence the braking assistance system can be initiated to assist the driver.
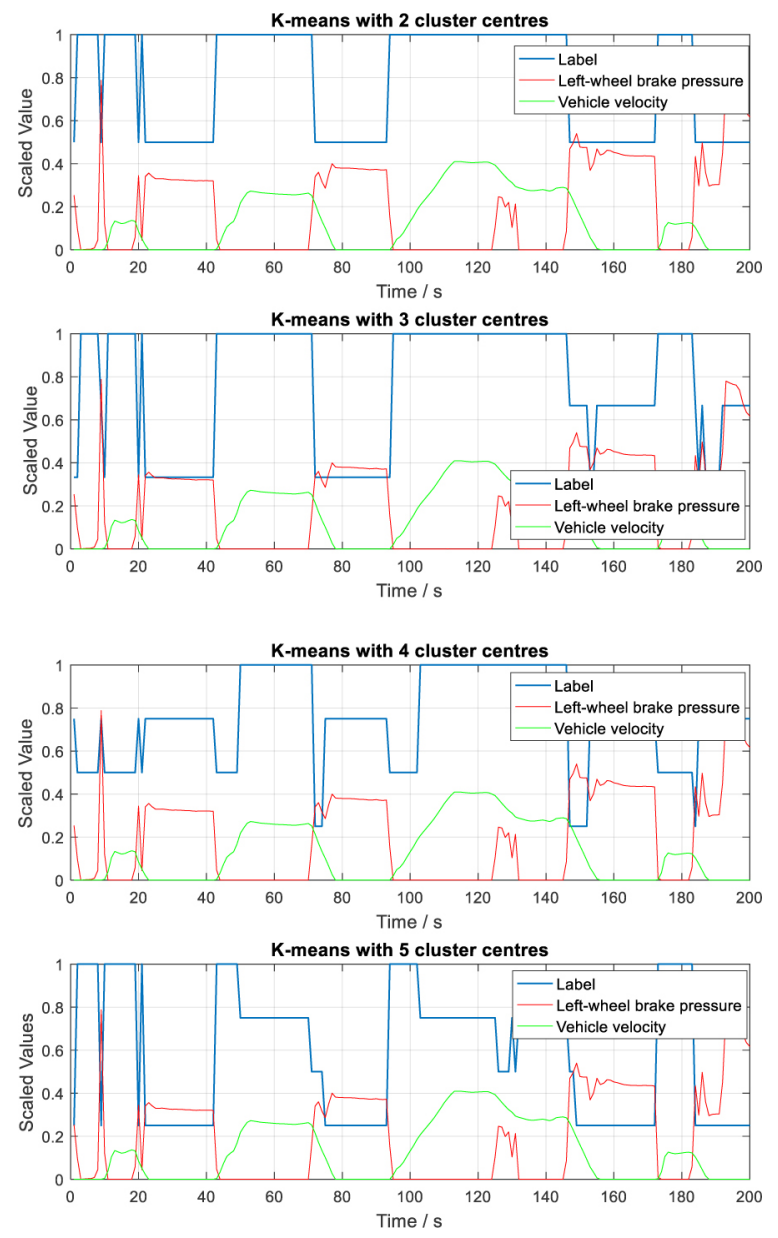

Figure 2. K-means clustering performance with different cluster numbers. From top left to bottom right is K-means algorithms with two to five cluster centers, respectively.

To verify the impact of different CAN bus signals to the clustering results, we evaluated the correlation coefficient between the input signals with the clustering results given by the two cluster centers scenario. The correlation coefficient is calculated with Pearson method to measure the linear dependence between two variable vectors. In this case, the correlation coefficient is calculated separately between the selected 12 CAN bus signals and the cluster label. The results located within the range of -1 and 1 , where 1 is total positive correlation, -1 is total negative correlation, and 0 means no correlation between each other. The correlation coefficient is calculated as:

$$
\rho_{X, Y}=\frac{\operatorname{cov}(X, Y)}{\sigma_{X} \sigma_{Y}}
$$

Where $\sigma_{X}, \sigma_{Y}$ are the standard deviation of variables X and Y. $\operatorname{cov}(X$, $Y$ ) is the covariance between $\mathrm{X}$ and $\mathrm{Y}$ which can be represented as:

$$
\operatorname{cov}(X, Y)=E\left[\left(X-\mu_{X}\right)\left(Y-\mu_{Y}\right)\right]
$$


Where $[E]$ and $\mu$ are expectation calculator and mean value respectively.

Table 4 below illustrates the relationship between different features and the clustering result.

Table 4. Correlation coefficients of the selected signals and K-means cluster result

\begin{tabular}{|c|c|c|c|}
\hline Signal No. & $\begin{array}{c}\text { Correlation } \\
\text { Coefficient }\end{array}$ & Signal No. & $\begin{array}{c}\text { Correlation } \\
\text { Coefficient }\end{array}$ \\
\hline 1 & -0.0229 & 7 & -0.7871 \\
\hline 2 & 0.6470 & 8 & -0.4003 \\
\hline 3 & 0.6470 & 9 & -0.7871 \\
\hline 4 & 0.6470 & 10 & -0.7871 \\
\hline 5 & 0.6470 & 11 & 0.3328 \\
\hline 6 & -0.7870 & 12 & -0.4947 \\
\hline
\end{tabular}

From Table 4 we can see that some of the signals has positive or negative strong influence to the cluster result while others have weak influence. Specifically, the wheel brake pressure signals give a significant contribution to the cluster performance as well as velocity information. Since there is a small difference between some similar signals, we can select fewer features to retrain the cluster and increase the computation efficiency of the system. Another interesting result is the acceleration pedal position (signal 8, correlation coefficient value -0.4003 ) is not highly correlated with the clustering result, therefore, we can also ignore this feature, which in the beginning we think it can be important.

\section{GMM Result}

Similar to the process shown in K-means, in this part, the data are clustered by GMM algorithms with different cluster number ranging from two to five and the correlation coefficients are also evaluated. The performance of GMM methods is illustrated in Figure 3 below. The cluster label is represented with blue line, left front brake pressure and vehicle velocity are shown in red line and green line respectively. Figure 3 is a short period of the driving cycle, which in the range between 0 second to 200 seconds like Figure 2.

From Figure 3 we can see that similar to K-means, GMM algorithm can also detect the braking intention accurately. When the braking maneuver occurs, GMM can choose the correct label in the very beginning. A detail clustering performance of GMM can be found in the Appendix.

Similarly, the correlation coefficient of GMM is proposed as it is used in K-means, the Pearson values given by different features are illustrated in Table 5 below.

From Table 5 it can be found that the most significant features for GMM clustering is as same as that in K-means. Specifically, the most important factors are brake pressures and the different kinds of velocity information.

Table 5. Correlation coefficients of the selected signals and GMM cluster result

\begin{tabular}{|c|c|c|c|}
\hline Signal No. & $\begin{array}{c}\text { Correlation } \\
\text { Coefficient }\end{array}$ & Signal No. & $\begin{array}{c}\text { Correlation } \\
\text { Coefficient }\end{array}$ \\
\hline 1 & 0.0355 & 7 & -0.5959 \\
\hline 2 & 0.8355 & 8 & -0.4128 \\
\hline 3 & 0.8352 & 9 & -0.5959 \\
\hline 4 & 0.8341 & 10 & -0.5959 \\
\hline 5 & 0.8337 & 11 & 0.2461 \\
\hline 6 & -0.5961 & 12 & -0.4584 \\
\hline
\end{tabular}
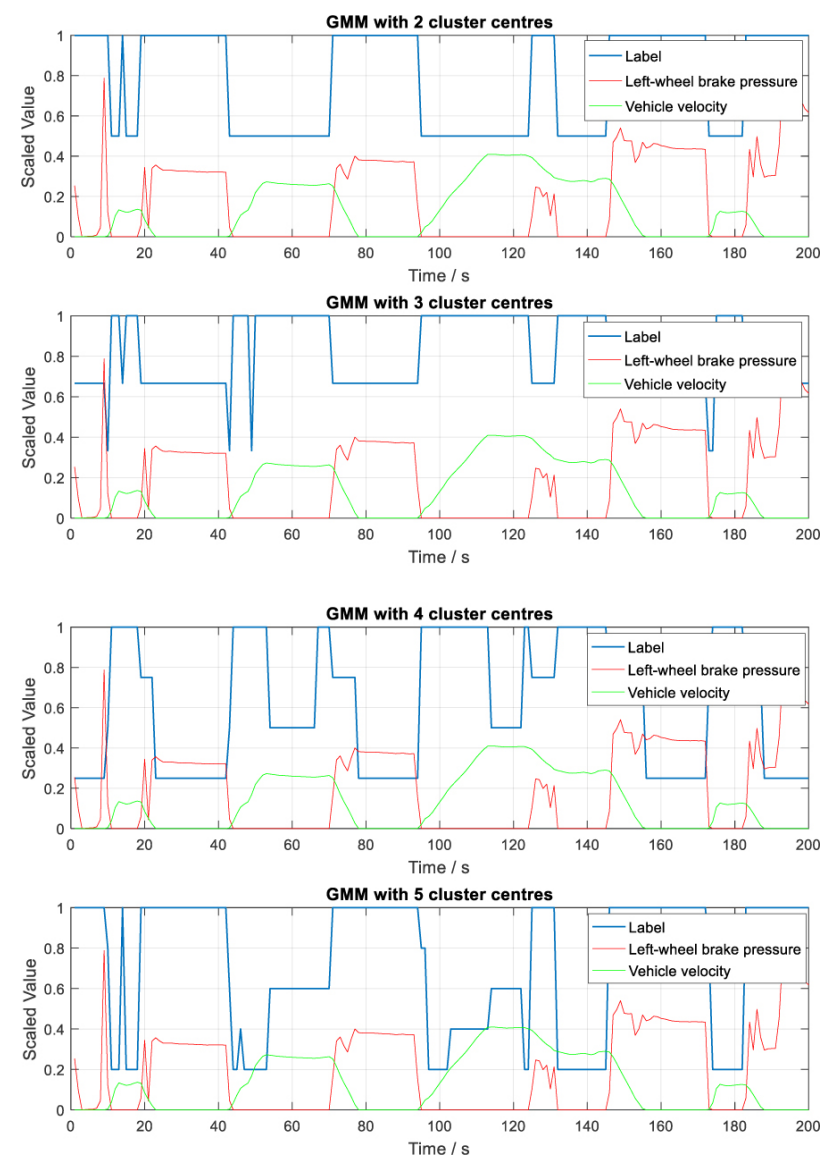

Figure 3. GMM clustering performance with different cluster numbers. From top to bottom is GMM algorithms with two to five cluster centers respectively.

\section{Discussion}

From Table 4 and Table 5 it can be seen that the key features for braking intention clustering are the wheel pressure values, vehicle velocity, and motor speed. Since some of these signals after re-scaled between zero and one has no big difference with each other, for example, vehicle velocity, motor speed and wheel speed equally contribute to the final result. Therefore, we can select one signal among them instead of using all of them. with consideration of computation efficiency, we select a subset of the above features and only left brake pressure and vehicle velocity are chosen as the cluster algorithm inputs. Figure 4 and $\underline{5}$ below gives the performance of K-means and GMM respectively with two input signals and two cluster centers.

Evaluation methods for braking intention can be divided into two scopes. The first one is prediction horizon which measures how much earlier the prediction make before the manoeuver start. Another method is to test the algorithm accuracy which check how many times it detects the braking process successfully. In this work, since we only use CAN bus information, we cannot predict the intention before driver takes any maneuvers. Therefore, we test the detection accuracy of the two algorithms and determine the specific braking moment during the driving cycle. A good performance for the algorithms is to recognize the braking process as accuracy as possible. 


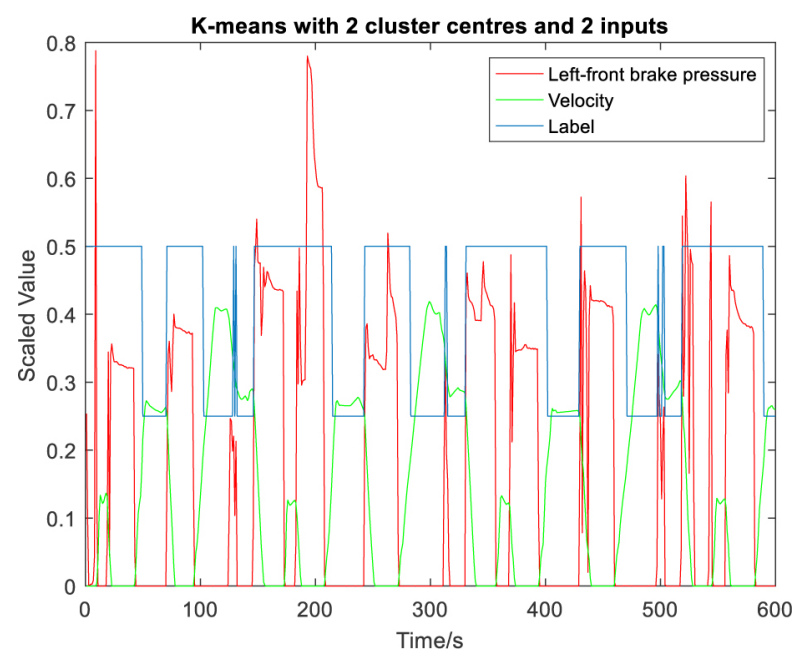

Figure 4. K-means clustering performance with 2 cluster centers and the selected 2 input signals in the first 600 seconds.

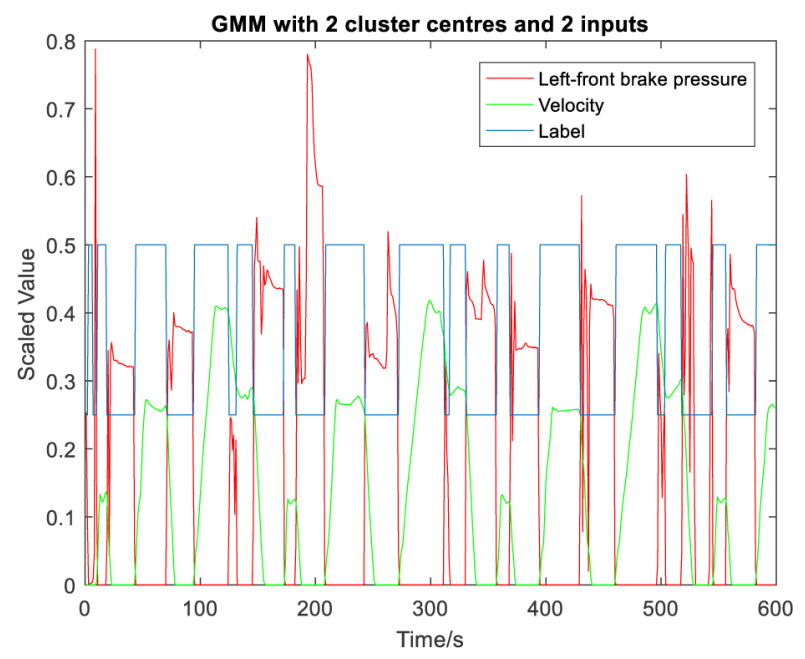

Figure 5. GMM clustering performance with 2 cluster centers and the selected 2 input signals in the first 600 seconds.

As can be seen from Figure 4 and Figure 5, both K-means and GMM with two most influential features (brake pressure and vehicle velocity) give a good performance in braking intention prediction. In addition, GMM gives a better and more sensitive recognition than K-means. For example, K-means cannot identify the small increase of velocity between 150 s and 250 s and give braking maintaining signal. However, in term of GMM, this small velocity increase can be detected and the system is more precise and sensitive to the input variation. In total, there are 15 times braking process between 1 and 600 seconds. GMM recognizes all the 15 braking process while $\mathrm{K}$-means only detects 8 times. Therefore, based on our data, the GMM performance overweigh K-means performance.

\section{Conclusions}

In this paper, driver braking intention recognition algorithm has been proposed with both K-means and Gaussian Mixture model. The reason of using unsupervised learning is the difficulty of determining the true moment when driver intention occur. The supervised knowledge (true moment or true label of intention) cannot be obtained with CAN bus signals only. The unsupervised learning methods automatically group the driving status into a few groups and identify braking process accordingly. This will further contribute to other classification works such as emergency/non-emergency braking classification. Unsupervised learning methods give a brief illustration of the rules hidden behind the driving cycle. The proposed test is based on a pure electric vehicle under EUDC driving cycle. 12 potential signals are selected as the inputs of cluster algorithm in the beginning, then with the calculation of correlation coefficient, the two most relevant features, left front brake pressure and vehicle velocity, are determined. Performances givens by K-means and GMM are compared. Final conclusion is made that both of the two algorithms can efficiently recognize driver braking intention at the very beginning moment. When the cluster label changes to braking group, it can be viewed as the driver is going to execute a period of braking maneuver so that braking assistance system can be turned on. In addition, the proposed result indicates that GMM is more sensitive and generates a more precise result than K-means algorithm.

\section{References}

1. Bareket Z, Fancher P S, Peng H, Methodology for assessing adaptive cruise control behavior. IEEE Transactions on Intelligent Transportation Systems, 2003, 4(3): 123-131.

2. Rong H, Gong J. Study on intelligent control of road condition perception and forward collision warning system. Intelligent Computing and Integrated Systems (ICISS), 2010 International Conference on. IEEE, 2010: 285-289.

3. Liu J F, Su Y F, Ko M K, Development of a vision-based driver assistance system with lane departure warning and forward collision warning functions. Digital Image Computing: Techniques and Applications (DICTA), 2008. IEEE, 2008: 480-485.

4. Qu, T., Chen, H., Cao, D., Guo, H., Gao, B. Switching-based stochastic model predictive control approach for modeling driver steering skill. IEEE Transactions on Intelligent Transportation Systems, 16(1): 365-375, 2015.

5. Crolla, D. A., Cao, D. The impact of hybrid and electric powertrains on vehicle dynamics, control systems and energy regeneration. Vehicle system dynamics, 50(sup1): 95-109, 2012.

6. Martinez, C.M., Hu, X., Cao, D., Energy Management in Plugin Hybrid Electric Vehicles: Recent Progress and a Connected Vehicles Perspective, IEEE Transactions on Vehicular Technology, in press, 2016.

7. Hu, X., Jiang, J., Egardt, B., Cao, D. Advanced power-source integration in hybrid electric vehicles: Multicriteria optimization approach. IEEE Transactions on Industrial Electronics, 62(12): 7847-7858, 2015.

8. Lv, C., Zhang, J., Li, Y. Extended-Kalman-filter-based regenerative and friction blended braking control for electric vehicle equipped with axle motor considering damping and elastic properties of electric powertrain. Vehicle System Dynamics, 2014, 52(11): 1372-1388.

9. Lv, C., Zhang, J., Li, Y., Yuan, Y. Mode-switching-based active control of powertrain system with nonlinear backlash and flexibility for electric vehicle during regenerative deceleration. Proceedings of the Institution of Mechanical Engineers Part D Journal of Automobile Engineering, 2015, 229(11): 1429-1442.

10. Zheng Y, Li S E, Wang J, Stability and scalability of homogeneous vehicular platoon: Study on the influence of information flow topologies[J]. IEEE Transactions on Intelligent Transportation Systems, 2016, 17(1): 14-26. 
11. Wang J, Zhang L, Zhang D, An adaptive longitudinal driving assistance system based on driver characteristics. IEEE Transactions on Intelligent Transportation Systems, 2013, 14(1): 1-12.

12. Enache N M, Mammar S, Glaser S, Driver assistance system for lane departure avoidance by steering and differential braking. IFAC Proceedings Volumes, 2010, 43(7): 471-476.

13. Zheng, H. and Ma, S., "Development and Test of Braking Intention Recognition Strategies for Commercial Vehicle," SAE Technical Paper 2015-01-2841, 2015, doi:10.4271/2015-01-2841.

14. Lopez A, Sherony R, Chien S, Analysis of the BrakingBehaviour in Pedestrian Automatic Emergency Braking. 2015 IEEE 18th International Conference on Intelligent Transportation Systems. IEEE, 2015: 1117-1122.

15. Aoude G S, Desaraju V R, Stephens L H, Driver behavior classification at intersections and validation on large naturalistic data set. IEEE Transactions on Intelligent Transportation Systems, 2012, 13(2): 724-736.

16. McCall J C, Trivedi M M. Human behavior based predictivebrake assistance. 2006 IEEE Intelligent Vehicles Symposium. IEEE, 2006: 8-12.

17. Wada T, Doi S, Nishiyama A, Analysis of braking behavior in car following and its application to driver assistance system. Proc. of International Symposium on Advanced Vehicle Control. 2008, 2: 577-583.

18. Haufe, S. Kim, J.-W. Kim, I.-H. Sonnleitner, A. Schrauf, M. Curio, and G. Blankertz, B. "Electrophysiology-based detection of emergency braking intention in real-world driving., " J. Neural Eng., vol. 11, no. 5, p. 056011, 2014.

19. Haufe, S. Treder, M. S. Gugler, M. F. Sagebaum, M. Curio, and G. Blankertz, B. "EEG potentials predict upcoming emergency brakings during simulated driving., "J. Neural Eng., vol. 8, no. 5, p. 056001, 2011.

20. Moon T K. The expectation-maximization algorithm. IEEE Signal processing magazine, 1996, 13(6): 47-60.

21. MacQueen J. Some methods for classification and analysis of multivariate observations[C]/Proceedings of the fifth Berkeley symposium on mathematical statistics and probability. 1967, 1(14): 281-297.
22. Everitt B S. Finite mixture distributions[M].John Wiley \& Sons, Ltd, 1981. Wiley.ISBN 0-471-90763-4.

23. Rasmussen C E. The infinite Gaussian mixture model[C]//NIPS. 1999, 12: 554-560

24. Hu X, Jiang J, Cao D, Battery health prognosis for electric vehicles using sample entropy and sparse Bayesian predictive modeling[J]. IEEE Transactions on Industrial Electronics, 2016, 63(4): 2645-2656.

25. Aoude G S, Desaraju V R, Stephens L H, Driver behavior classification at intersections and validation on large naturalistic data set. IEEE Transactions on Intelligent Transportation Systems, 2012, 13(2): 724-736.

\section{Contact Information}

Dr. Yang Xing

Advanced Vehicle Engineering Center, Cranfield University

Cranfiled, MK43 0AL, United Kingdom

y.xing@cranfield.ac.uk

Dr. Lv Chen

Advanced Vehicle Engineering Center, Cranfield University Cranfiled, MK43 0AL, United Kingdom

C.Lyu@.cranfield.ac.uk

Dr. Huaji Wang

Advanced Vehicle Engineering Center, Cranfield University Cranfiled, MK43 0AL, United Kingdom

Huaji.Wang@cranfield.ac.uk

Dr. Wang, Hong

Department of Mechanical and Mechatronics Engineering, University of Waterloo, Canada

h492wang@uwaterloo.ca

Dr. Dongpu Cao

Advanced Vehicle Engineering Center, Cranfield University Cranfiled, MK43 0AL, United Kingdom

D.cao@cranfield.ac.uk 


\section{$\underline{\text { APPENDIX }}$}
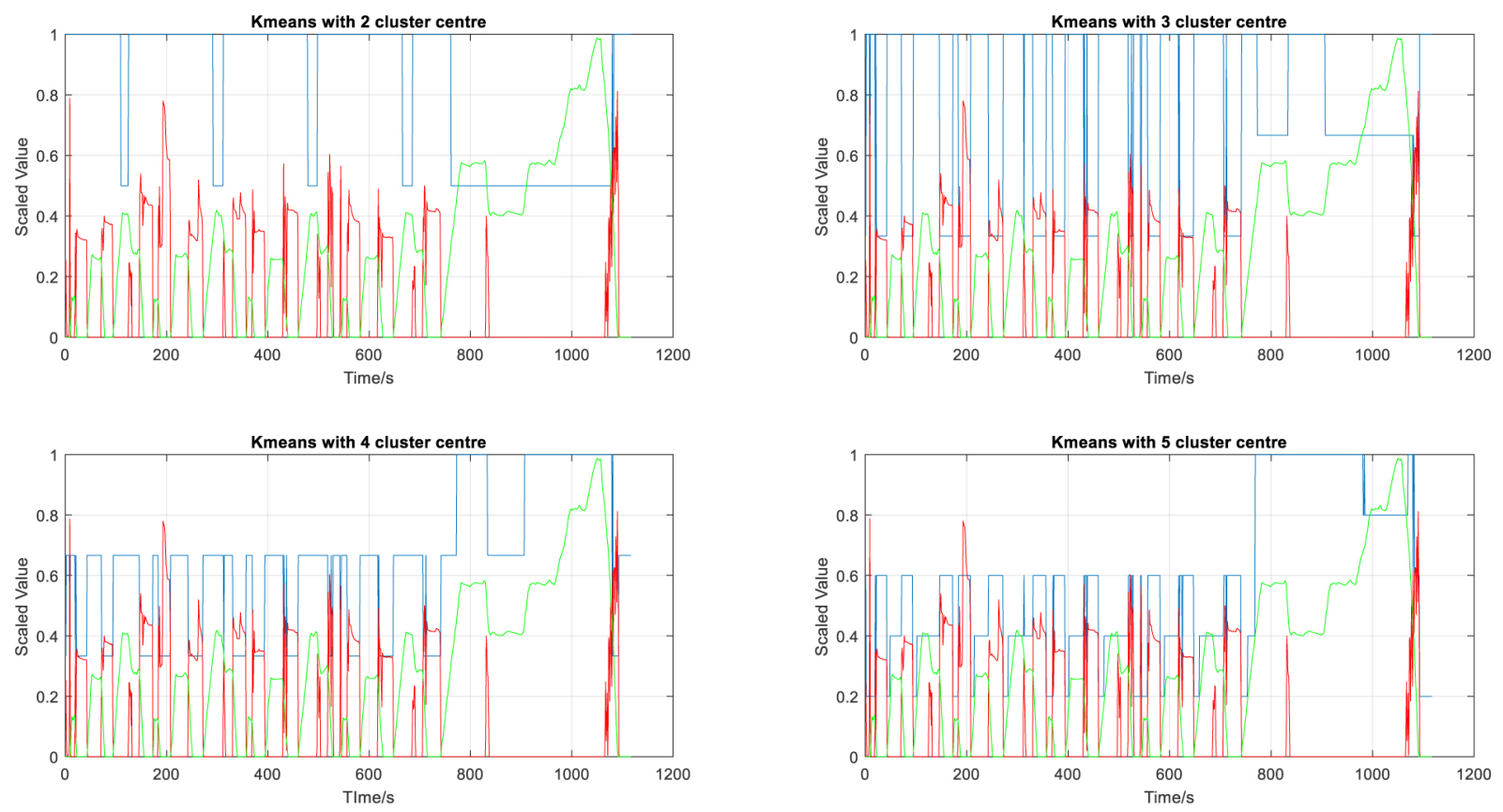

Figure 1. K-means clustering methods with cluster center number ranging from 2 to 4 of the total driving cycle.
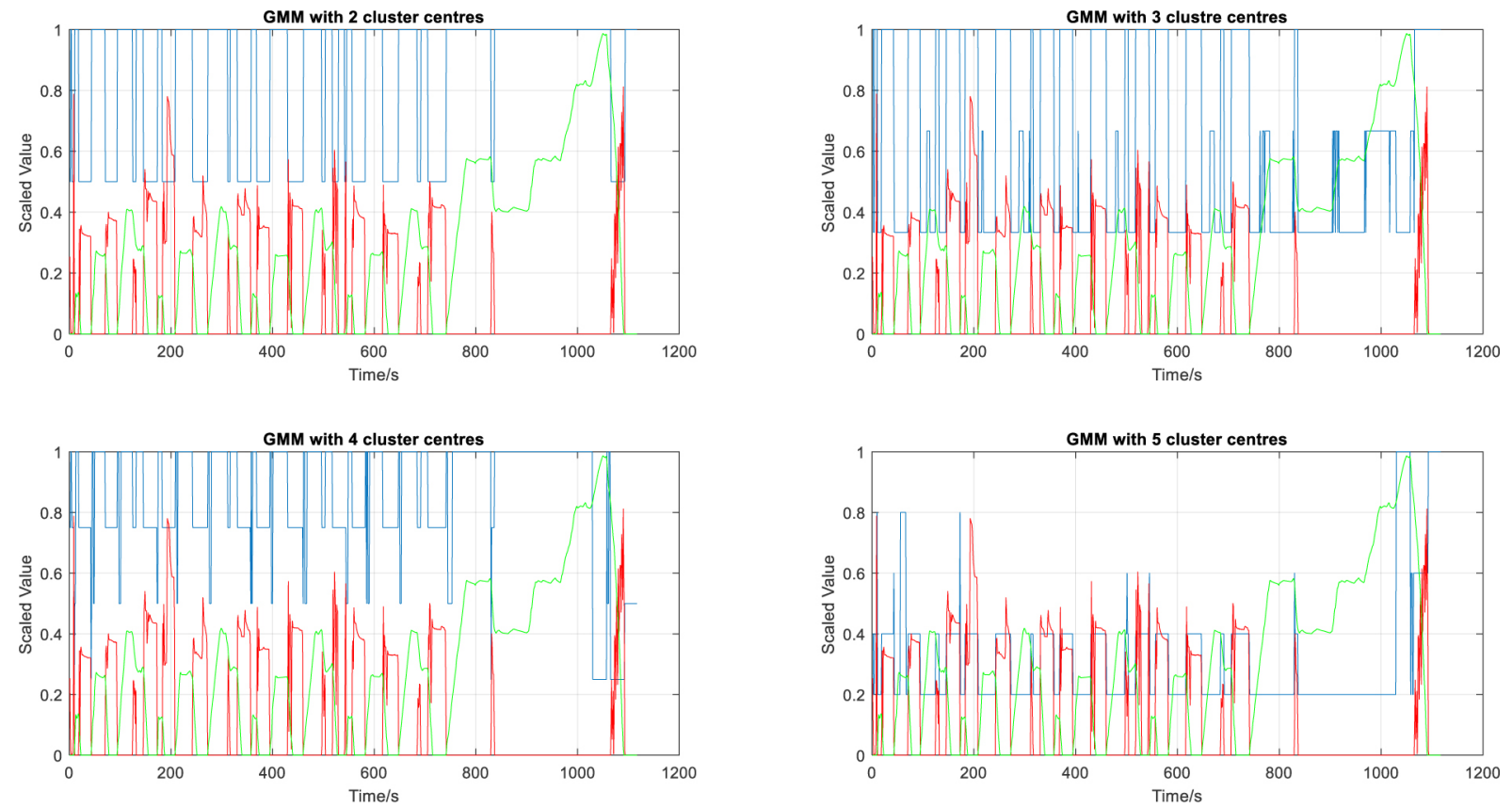

Figure 2. Gaussian Mixture Model clustering methods with cluster center number ranging from 2 to 4 of the total driving cycle.

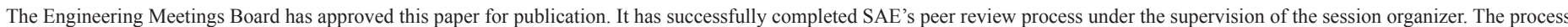
requires a minimum of three (3) reviews by industry experts.

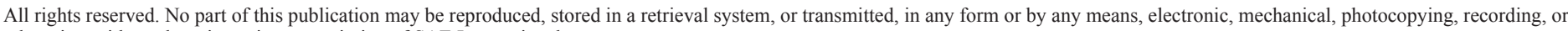
otherwise, without the prior written permission of SAE International.

Positions and opinions advanced in this paper are those of the author(s) and not necessarily those of SAE International. The author is solely responsible for the content of the paper.

ISSN 0148-7191

http://papers.sae.org/2017-01-0433 
2017-03

\section{Recognizing driver braking intention with vehicle data using unsupervised learning methods}

Xing, Yang

SAE International

Xing Y, Lv C, Huaji W, Wang H, Cao D, Recognizing driver braking intention with vehicle data using unsupervised learning methods, WCX17: SAE World Congress Experience, Detroit, 04/04/2017 - 06/04/2017. DOI: 10.4271/2017-01-0433

https://saemobilus.sae.org/content/2017-01-0433

Downloaded from Cranfield Library Services E-Repository 